$x^{2}$

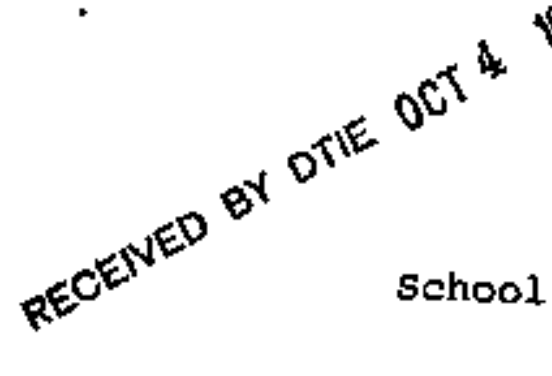

98

$$
\cdots
$$

\title{
University of Minnesota
}

of Physics, Institute of Technology

CESTI REICES

He. 3.10

Mr.

Experimental and Theoretical

Studies in solid state and Low Temperature Physics

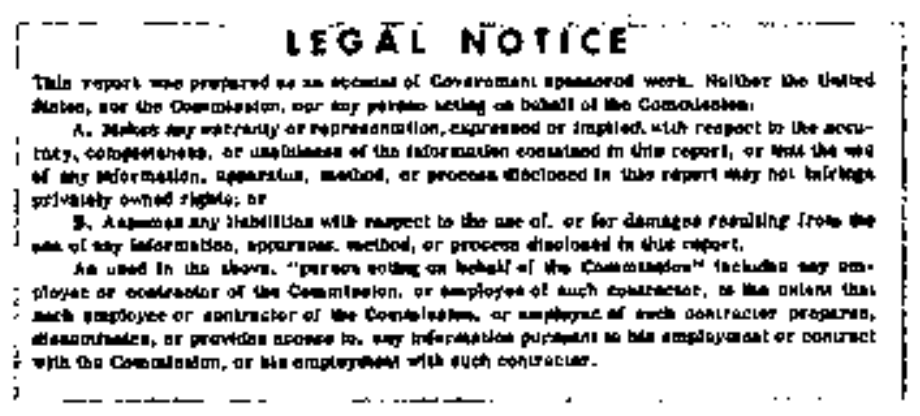

\section{ANNUAL - PROGRESS REPORT}

forr

$-1967$

Submitted

September, 1967

U.S. Atónic Energy Comnission Contract No. AT(i1-1)-1569 


\section{DISCLAIMER}

This report was prepared as an account of work sponsored by an agency of the United States Government. Neither the United States Government nor any agency Thereot, nor any of their employees, makes any warranty, express or implied, or assumes any legal liability or responsibility for the accuracy, completeness, or usefulness of any information, apparatus, product, or process disclosed, or represents that its use would not infringe privately owned rights. Reference herein to any specific commercial product, process, or service by trade name, trademark, manufacturer, or otherwise does not necessarily constitute or imply its endorsement, recommendation, or favoring by the United States Government or any agency thereof. The views and opinions of authors expressed herein do not necessarlly state or reflect those of the United States Government or any agency thereof. 


\section{DISCLAIMER}

Portions of this document may be illegible in electronic image products. Images are produced from the best available original document. 
TABLE OF CONTENTS

Page

I. INTRODUCTION

II. DESCRIPTION OF RESEARCH 2

A. superconductivity 2

1. The Josephaon Effect 2

a. Meissner Effect and Vortex Penetration in Wide, High-Current Jog̣ephaon Junction:

b. Stability of Metastable Current states of superconducting nings Interrupteò by Josephson Junctions

2. Quantum Effects Near the Superconducting Trandition Temperature

a. Extensions of the Little-Parks Experiment

b. Deflase-van Alphen Orcillationg in the

Critical Temperature of TYpe-II

Superconductors

B. Theory of Quantum Cryatels 7

1. Improved correlation Functions for 7 Quantum Crystals

2. Colculation of Phonon Frequemcies and B Thermodynatic Propertiea of Cryatalline BCC He.

3. A Critique of Saunder's Thoory of solid ${ }^{3} \mathrm{He}$ at $0^{\circ} \mathrm{K}$. 
4. Simple Approach to the Self-Consistent

Phonon Theory

5. Exchange in crystalline Helium Three

6. Study of the Nuclear Relaxation of the spin System of crystalline ${ }^{3} \mathrm{He}$

c. Mixtures of Helium Isotopes

1. Phase Separation in Liquid ${ }^{3} \mathrm{He}-{ }^{4} \mathrm{He}$ Mixtures

2. Phase separation of ${ }^{3}$ He- ${ }^{4}$ He crystals

D. Magnetism in Metals

1. Nuclear Magnetic Resonance in Magnetic Materials

2. Nuclear Orientation Experiments

E. Superfluidity in Liquid Helium

1. Experiments on Ion Trapping in Rotating Helium II 14

2. Experiments on Quantization of Superfluid circulation 16

3. Studies of superfluid Flow in a Porous Hediur 17

4. Other Work 18

F. Millidegree Facility 19

1. Adiabatic Demagnetization Cryostat 19

2. ${ }^{3} \mathrm{He}-{ }^{4}$ He Dilution Refrigerator 19

G. Properties of Liquid Helium near the Lambda Transition 20

1. Critical Indices of the Lambda Transition ${ }^{-} 20$

2. Liquid Helium Green Functions Near the Lambda Point 20 $\begin{array}{lr}\text { III. PERSONHEL } & \mathbf{2 1}\end{array}$

$\begin{array}{lr}\text { IV. BIBLIOGRAPHY } & 23\end{array}$ 


\section{I - INIRODUCTION}

The work described in this progress report consists of various experimental and theoretical investigations in a braad area which may be called Solid state and Low Temperature Physics. The research was under the direction of Profeasors A. M. Goldman, L. H. Nosanow, W. $V$. Weyhmann and $W$. Zirmermann at the school of Physics and Astronomy in the Institute of Technelogy of the University of Minnesota and was suppported by USAEC contract AT(11-1)-1569.

The reader is cautioned that results presented here are tentative and subject to modification prior to publication. 


\section{DESCRIPTION OF RESEARCH}

\section{A. Superconductivity}

\section{The Josephson Effect}

a . Meisgner Effect and Vortex Penetration, in Wide, HighCurrent Jogephson Junctions

Investigation of the magnetic fleld dependence of the maximum zero-voltage current of wide, high-current Josephson Junctions has revealed behavior drastically different from the usual Fraunhofer pattern for narrow junctions. 1 The experiments are interpreted as evidence for a Meissnex Effect for the insulating layer and adjacent penetration layers of wide junctions in low external fields and for an eventual transition to a mixed state ${ }^{2}$ as the external field is increased from zero. The observed current-field curves are in reasonable agreement with detailed calculations by owen and scalapino ${ }^{3}$. This work was reported at the March meeting of the American Physical Society 4 and will be published in The Physical Review ${ }^{4}$

1. J. M. Rowell, Phys . Rev. Letters 11, 200 (1963).

2. B. D. Josephson, Adv. in Physics 14, 419 (1965).

3. D. J. Scalapino and C. J. Owen (to be published).

4. A. M. Goldman ana P. J. Kreisman, Bull. Am. Phys. Soc. II 12, 309 (1967)

5. A. M. Goldman and P. J. Kreisman, Phys. Rev., to be published, USAEC Report c00-1569-10. 
b. Stability of gurrent states of superconducting Rings Interrupted by Josephson Junctions

The experimenta of Goldman, Kreiman, and scalapino ' have been continued and extended. In this work circulating ring currents in superconducting rings containing two junctions were detected by measuring the depression of the maximum zero-voltage current through a parallel combination of the two junctions. Up to ten quantum levels have been observed in recent experiments. The various levels were randonly populated by repeatedly traversing the I-V characteristic of the ring atructure. Histograms of the occurrence of the various states were recorded digitally. Measurements of the temperature dependence of the level structure were also made. Ring currents were also prepared by warming and cooling in the presence of an axial magnetic field and removing the field. A single meaurement of the maximum zero-voltage current then could be used to destructively read out the ring current. Results of these experinents appear to be in gowd agreement with theory. A detailed description of this wark will shortly be submitted to The Phygical Review. A brielf account will appear in the proceedings of the Tenth. International Conference on Low Temperature Physics. 2

Development of a flux-flow magnetometer ${ }^{3}$ to be used to extend this work is well underway. 
1. A. M. Goldman, P. J. Kreisman and D. J. Scalapino, Phys. Rev. Letters 15,495 (1965).

2. A. M. Goldman and P. J. Kreisman, Proceedings of the Tenth International Conference on Low Temperature Physics, Moscow 1966 (in press), USAEC Report no. c00-1569-5.

3. J. E. Méccereau, Proceedings of the Conference on Superconducting Devices, charlottesville 1967 (in presg). 
2. Quantum Effects at the superconqucting Transition Temperature

(A. M. Goldman)

a. Extengions of the Little-Parks Experiment

Experiments to observe the periodicity of the transition temperature of long superconducting rings in axial applied magnetic fields were initiated during the past year. This work has two aigst the first is to-cfieck the observation of Little and Parks of an apparent helBe flux period for indium in addition to the usual he/2e value. The second is to search for the predicted large depression of the transition temperature of low inductance cylinders caused by depairing due to induced ring currents. These investigations are current ly: be ing pursued using both circular and planar ringt.

'1. W. A. Little and R. D. Parks, Phys, Rev. 133, A97 (1964).

2. Peter Fulde and R. A. Ferrell, Phys. Rev. 131, 2457 (1963).

b. De Haas-van Alphen Oscillations in the critical Temperature of Type-II superconductors

It was recentiy predicted ${ }^{i, 2}$ that the critical temperature of a Type-II superconductor should exhibit de"Hasmivan łaphen like oscillations. The theory predicts the ratio of the amplitude of the temperature oscillations to the critical temperature to be $10^{-4}$ at $0.025^{\circ} \mathrm{K}$ and -

A dilution refrigerator, which will be uged to reach the required temperature range is being assembled. A sensitive quantum voltmeter which employs a solder bead on a niobium wire ${ }^{3}$ has already been con- 
structed. The latter device is capable of resolving $10^{-14}$ volts. samples of high purity niobium with resistivity ratio in excest of 10,000 have been made available to us by the Union carbide Corporation for this experiment.

1. Leon Gunther and Leonard W. Gruenberg, Solid State Communications 4, $329(1966)$.

2. A. K. Rajagopal and R. Vasudervan, Physics Letters 23, 539 (1966). 3. J. Clarke, Phil. Mag. 13, 115 (1966). 
B. Theory of Quantiam crystals

1. Improved correlation Functions for ouantum Crystals (W. J. Mu111n, L. H. Nosanow and E. M. sqteinback)

In recent work ${ }^{1}$, it has been shown that the cluster expansion approach leads to a reasonable approximation-scheme for calculating the properties of quantum crystals, at least for a restricted class of correlation functions. An obvious question which has been opened by this work is whether it is possible to improve the theory by the use of better correlation functiợ. This problem has been studied by using the cluster expansion approximation for the ground-state energy and writing a differential equation for. correlation function. It has been found necessary to include the 3-body terms in the differential equation and to impose a constraint on the long-range behavior of the correlation function, since this behavior is not determined by the leading terms of the cluster expansion. The computer program to solve this differential equation has been written and is currently being debugged.

1. J. H. Hetherington, H. J. Mullin and L. H. Nosanow, Ehys. Rev. 154, 175 (1967). 
2. Calculation of Phonon Frecuencies and Thermodymamic Properties of crystalline $B C C$ He (F. W. de wette (University of Texas), L. H. Nosanow and N. R. Werthamer (Bell Telephone Laboratories;) ).

In this paper the lattice dynamics of crystalline bcc helium is treated by using the time-dependent. Hartree approximation together with the results of variational calculations of the ground-gtate energy using correlated trial wave functions. The phonon spectrum has been calculated for various densities of bce $\mathrm{He}^{3}$ and $\mathrm{He}^{4}$. We present dispersion clarves, density of state histograms and sound velocities for selected densities. In addition we have calculated (with these densities) the mean displacement and specific heat. This paper has been submitted for publication in The Physical Review.

3. A criticue of The Saunders Theory of solid $\mathrm{He}^{3}$ at $0^{\circ} \mathrm{K}$ (W. J. Mullin)

An examination is made of the theory of the ground-state of solid ${ }^{3} \mathrm{He}$ proposed by saunders ${ }^{1}$ and modified by Garwin and Iandesman. ${ }^{2}$. We find the theory is inconsistent or wrong in the following respects: unphysical, single-particle functions result from misapplication of the Pluvinage ${ }^{3}$ method of generating wave function; the derivation of -an expression for the exchange inţegral $J$ is inconsistent with. this wave function; a correct solution for the integral equation for the single-particle probability density has not been given; the beuristic expression used for thercohesive energy is inconsigtent with the Pluvinage method. This paper has been submitted for publication in The Physical Review. 
1. . . M. Saunders, Phys a Reva 126, 1724 (1962).

2. R. L. Garwin and A. Landesman, Physics 2, 107 (1965).

3. P. Pluvinage, Ann. Phys, $\underline{5}, 145$ (1950).

4. Simple Approach to the Self-Consistent Rhonon Theory (L. H. Nosanow)

In the last year the use of self-consistent phonons has been shown to be important not only for quantum crystals, but also for other noble gas crystale. The existing derivations of this approach by Koehler ${ }^{1}$ or Horner ${ }^{2}$ are rather compdicated. It is possible to give a simpler derivation of this approach by studying the equation of motion. one finds that these equations can be recast in form such that the leading term is the self-consistent harmonic approximation and that succeasive terms yield corrections which may be identified as interactions between these self-consistent phonons. A paper on this work is in preparation.

1. T. M. Koehler, Phys. Rev, Letters 18, 654 (1967).

2. H. Horner, Z.. Physik, to be published.

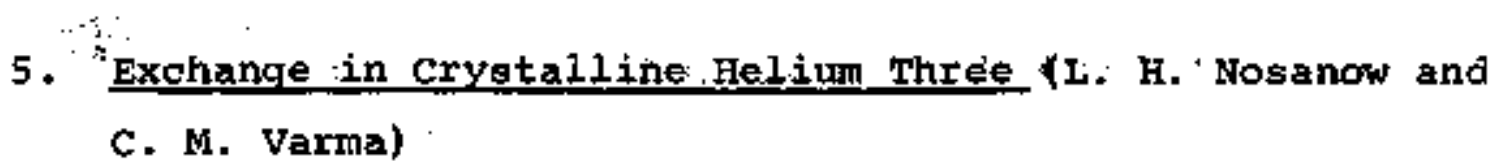

A derivation of the appropriate apin Hamiltonian for the nuclear spin system of cxygtalline helium three has been given. It is found that the exchange integral must be replaced by an appropriately defined exchange operator. I: Exptessions for ind operator have been given within the context of the cluster 
expansion. The temperature dependence of the axchange frequency is studied within the context of this approsch. A report of this work will be submitted to Physical Reyiew Letters.

6. Study of tho Nuclear Relaxation of the Spin Sygten of crystalline ${ }^{3}$ He (L. H. Nosanow and C. M. Varma)

The various mechaniams for the relaxation of the nuclear spin system of crystalline helium three are extremely complicated. This work is part of a systematic study of all of the nuclear relaxation mechanisms of this system. The main progress so far has been made with respect to the relaxation of the system from the exchange bath to the lattice which can be caused by the lattice modulation of the dipole interaction. The equations for the relaxation rate due to this process have been derived using the results of the theory of quantua crystals and include the effects of self-consistent phonons. The numerical cafculation of this relaxation rate is in progress. An interesting physical feature is that there is a lower bound to this rate which is determined by the very large zero-point motion in crystalline helium. Thus there is an intrinsic upper limit to the relaxation time which is set by the zero-point motion. consequently crystalline ${ }^{3}$ He might be the best subatance to use for cooling by nuclear demagnetization. 
c. Hixtures of Helium Isotopes

1. Phase Separation in Liquid ${ }^{3}$ He- ${ }^{4}$ He Mixtures

(R. D. Murphy and I. B. Nosanow)

The ground state properties of liquid ${ }^{3} \mathrm{He}-{ }^{4} \mathrm{He}$ mixtures have been studied. The approach is to introduce the experimental information on the pure isotopes and thereby obtain information about the mixtures of the isotopes, $i$.e. we are trying to find an interpolation procedure which will give the concentration dependence of various properties of the mixture. It is hoped that this work will yield accurate raculations of quantities like the partial molar volume of ${ }^{3} \mathrm{He}$ and ${ }^{4} \mathrm{He}$ which are very important from a theoretical point of view. calculations using this approach are currently in progress.

2. Phase Separation in ${ }^{3} \mathrm{He}-{ }^{4}$ He Crystals ( $\% . J . M u l l i n$ )

The calculations which have been used to study the properties of crystals of pure ${ }^{3}$ He and ${ }^{4}$ He have been extended to treat srystalline mixtures of these isotopes. It has been assumed that the different isotopes are distributed randomly. with this assumption, it is possible to calculate the ground-state energy in a straightforward mannex. The effects of finite temperatures have also been included in a simple way. With this theory it is possible to calculate the phase separation curve for ${ }^{3} \mathrm{He}-{ }^{4} \mathrm{He}$ crystal mixture. Areliminary results indicate qualitative agreement with experiment, although the phase separation temperature is off by a factor of two. A particularly encouraging feature of the calculation is that the phase separation curve has the correct form at very low concentra- 
D. Magnetisn in Metals

1. Nuclear Resonance in Magnetic Materials (R. Houghton, J. Aslam, J. Waner, w. Weyhmann)

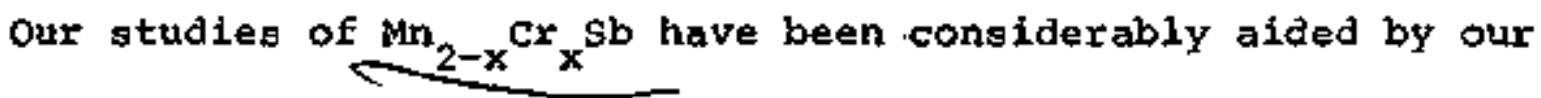
completed development of a reliable and versatile spin-echo spectrometer which is simple to use and free of long-line problems. A single set of instraments is used from 50 to $1000 \mathrm{MHz}$. This range can easily be extended if necessary. Work from liquid helium temperatures to well above room temperature is feasible, though we have only used the instrument from 55 to $300^{\circ} \mathrm{K}$.

Most of our resonance work on the Mn-Sb gystems has been centered on pure $\mathrm{Mn}_{2} \mathrm{sb}$ in the vicinity of the spin-flip temperature $\left(240^{\circ} \mathrm{K}\right)$. Spin echoes from both of the manganese sites and the antimony site have been observed throughout this region. For the manganese resonances, the quadrupole interaction is washed out in the spin flip region but is observable some $20^{\circ} \mathrm{K}$ on either side of $240^{\circ} \mathrm{K}$. The nuclear spin relaxation times, $\mathrm{T}_{1}$ and $\mathrm{T}_{2}$, have been measured within the range of temperatureg from $77^{\circ} \mathrm{K}$ to $300^{\circ} \mathrm{K}$ which includesi the spin flip region as rexpeted, $\mathbf{T}_{1}$ decreases montonically with increasing temperature and $\mathbf{T}_{2}$ also decripases montonically with increasing temperature except in the spin flip region. There it exhibits a minimum and then a maximum at the spin flip temperature; above $240^{\circ} \mathrm{K}$ it resumes its monotonic decrease with increasing temperature.

A tunnel diode oscillator operable at cryogenic temperatures has been developed. Its sensitivity is hard to control, but the device is still 
valuable for wide-frequency-range searches and for measuring the temperature dependence of the resonance frequency of obsefved 1 ines. It is being used to search for the resonance of various impurities in iron, cobalt, and nickel.

We have also constructed a radio-frequency induction furnace for preparing high purity samples under vpouum. This apparatus was essential. for the preparation of the iron alloys.

2. Nuclear orientation Experiments (C. Smith and $w$. Weyhmann)

Construction of the basic components of the adiabatic demagnetization cryostat was completed in January and assembly of the apparatus was (n)

completed about'two months later. The basic refrigeration stages to $0.3^{\circ} \mathrm{K}$ now operate adequately. The demagnetization magnet (obtained from westinghouse) has been tested. A lead heat switch driven by the magnet is being optimized. Installation of a salt' pill and sample should be completed by the end of Semptember.

The associated nuclear counting equipment is ready and the analyzer is operative. An incremental magnetic tape recorder will be used to read out the information. This tape can be directly put on the computer and is an infinite improvement over the punched tape originally proposed in speed, reliability and ultimate cost. 
E. Superfluidity in Liquid Helium

1. Experiments on Ion Trapping in kotating Helium -II (W. Zimmermann, Jr。 and $W$. Pratt)

;

The lifetime for the escape of regative ions from quantum vortices in rotating superfluid heliuf has been measured as a function of teruperature both at saturated vapor pressure and at elevated pressures up to the solidfication pressure. The method used was suitable for measuring lifetimes in the range from about $10 \mathrm{sec}$ to $1000 \mathrm{sec}$, and measurements were made within the range of temperatures from $1.09^{\circ} \mathrm{K}$ to $1.67^{\circ} \mathrm{K}$. These measurements represent an extension to elevated pressures of the experiments of $R$. I. Douglass at saturated vapor pressure, and the results at saturated vapor pressure are in good agreement with his findings. Oux work complements the recent experiments of B. E. Springett and R. J. Donnelly on the capture cross section for negative ions in the rotating iliquid under pressure. ${ }^{2}$ We have analyzed our resulta in terms of the bubble model of the negative ion and have found a bubble radius of about 19 or $20 \AA$ at saturated vapor pressure decreasing to 12 or $13 \AA$ at the solidification pressure. Although somewhat larger than previous estimates, our values for the radius are in reasonably good agreement with the predictions of an extremely simple model for the bubble and are somewhat smaller than a recent experimental determination of the radius at saturated vapor pressure by J. A. Northby and T, M. Sanders. ${ }^{3}$

A report on this work was given at the fashington meeting of the A.P.S., April 24-27, $1967 .^{4}$ A detailed, report of this work has been prepared by w. P. Pratt as his Ph.D. Thesisf; and it is planned to 
submit an article on this work for publication in The Physical Review in the near future.

1. R. I. Douglass, Rhys. Rev, Letters 13,791 (1964).

2. B. E. Springett, Phys. Rev. 155, 139 (1967).

3. J. A. Northby and T. M. Sanders Jx., Phys。 Rev, Letters 18, 1184 ,

4. W. P. Pratt and w. Zimmermann, Jra, Burll. Am, Phys. Soc. 12, 551 (1967), AEC Report C00-1569-9.

5. W. P. Pratt, Ph, D. Thesis, University of Minnesota (1967), AEC 'Report c00-1569-14) 


\section{Experiments on ouantization of superfluid circulation}

Our experimentg making use of the Vinen method to study quantization of superfluid circulation around a fine wire at saturated vapor pressure between $1.2^{\circ} \mathrm{K}$ and $1.9^{\circ} \mathrm{K}$ have been completed. ${ }^{1}$ The experiments represent an expension of Vinen's original work' in several ways, and have revealed stable superfluid circulations at the levels of 1,2 , and 3 quantum units. A detailed report of these experiments has been prepared by $s$. C. Whitmore as his Ph.D. thesis, ${ }^{3}$ and an article to be subritted to The Physical Review on this work has just been completed. 4

An effort to extend our work along these-lines is currently underway. The design of a new apparatus has almost been completed. This apparatus will permit the sample cell to be cooled to $0.4^{\circ} \mathrm{K}$ or below in order to avoid more completely the effects of the normal component of helium II. Provision has been made for pressurizing the sample, in particular, to permit the rotating state of helium II to be reached from the colid state. In addition, the apparatus has been designed to allow measurements to be made in rotation at higher speeds than was possible with the present apparatus.

1. S. C. Whitmore and $w$. Zimmermann, Jr., Phys. Rev, Letters 15, 389 (1965) .

2. W. F. Vinen, Proc. Roy. Soc. (Eondon) A260, 218 (1961).

3. S. C. Whitmore, Ph.D. Thesis, University of Minnesota (1966).

4. S. C. Whitmore and W. Zimmermann.p Jr., AEC : Report C00-1569-13. 


\section{Studies of superfluid Flow in a Poxous Medium}

our experiments studying the flow of the superfluid in powderfilled glass spheres has been completed. In these experiments studies were made both of the torsional oscillation of the spheres and also of the behavior of persigtent circulating superfluid flow in the spheresusing a gyroscopic technique.. A detailed report of this work was prepared by J. B. Mehl as his Ph.D. thesis, ${ }^{2}$ and an article on this work has been subratted to The Physical Review For publication. ${ }^{3}$

New experiments liavè been undertaken' to study superfluid flow in very fine channels. A superfluid gyro has been constructed for this purpose in the form of a powder-filled torus, with the flow circuit around the torus interrupted by a number of barriers of Millipore filter having pores on the order of $100 \%$ in size. It is, expected that the lowering of the $\lambda$-tiansition temperature in the barrier will reflect inself in the dependence at the angular monenturn of a persistent current on temperature, and that this experiment will provide a way for studying the variation in $\lambda$-point depresion as the pore size is varied.

In preliminary runs it has been found that the critical flow velocities in the filter appear to be surprisingly low and temperaturedependent. As a result we have considered it advisable to improve the sensitivity of our measuring sshenie before paking further measurments and a servo systera for controlling the gyro torsion suspension has been developed. 
1. J. B. Mehl and W. Zimmermann; Jr。, Phys. Rev. Letters 14, 815 (1965).

2. J. B. Mehl, Ph.D. Thesis, University of Minnesota (1966), AEC Report C00-1569-4.

3. J. B. Wehl and $W$. Zimmermann, Jr., AEC Report C00-1569-12.

\section{Other Hork}

a. Studies have been undertaicen to explore the feasibility of using tunnel cathodes to inject negativer ions into lidiulid helium. A highripged vacuum system has been assembled in which to carry out the evaporations needed to produce the cathodes.

b. Design studies have been carried out for an apparatus to study possible Josephson effect analogs? in the flow of superfluid helium through small orifice. Attention has been focused on the use of small capacitor microphones for both setting the fluid. into motion and detecting the small pressure differences that are expected to be of interest. For the latter an fm detection scheme is being considered. 
F. Millidegree Facility (A. M. Goldman, w. Weyhmann and W. Zimmermann, Jr.)

Significant strides towards the development of a coherent millidegree program have been made in the past year. The firgt stage of this program involves development of apparatus for reaching temperatures in the $0.100^{\circ} \mathrm{K}$ to $0.010^{\circ} \mathrm{K}$ range. To this end both an adiabatic demagnetization apparatus and a ${ }^{3}$ He- ${ }^{4}$ He dilution refrigerator are being constructed. The former is the customary meang of cooling systerms into the $0.100^{\circ} \mathrm{K}$ $0.010^{\circ} \mathrm{K}$ temperature range and the latter an extremely promising new technique. The adiabatic deroggnetization apparatus under construction will be employed in nuclear orientation studies (see Section D).

\section{Adabatic Demagnetization Cxyostat (W. Weyhmann)}

This apparatus is described in the section on nuclear orientation experiments .

2. ${ }^{3}$ He- ${ }^{4}$ He Dilution Refrigerator (A. M. Goldman and "A. Menaxd)

All of the equipment for the refrigerator has been purchased. The low temperature apparatus has been assembled and leak cheoked. A stokes 4" ring-jet pooster puxp_and a sealed Edwards $17 \mathrm{cfm}$ mechanical pump have been obtained for circulating ${ }^{3}$ He. sintered copper blocks have been fabricated. for use in the heat exchangex. Tha apparatus that has been constructed will be mounted in the bottom access dewar which was obtained from Sulfrian cryogenies corp. Trials should commence in late summer, 1967, upon corpletion of the gas handling system. 
G. Theoretical Properties of Liquid Elelium Near the Lambda Transition

\section{Critical Indices of the Lambda Transition}

The gidor-Kadanoff Scaling Laws are derived assuming that the free energy is an analytic function of the temperature and theoroder parameter except the transition point. Using this formulation ana microscopic theory we argue for an additional relation for the critical indices of the liguid helium $\lambda$-transition:

This work has been submitted to Physics Letterg,

2. Liguia Hei ium Green Functions near the Iambda Transition

The renormalized expressions for the thermodynamic potentials and the Green functions of an interacting Bose system have been studied in the region of small condensate density.

An account of this work has been submitted to The Physical Review. 2

1. Eero Byckling, USAEC Report c00-1569-8.

2. Eero. Byckling, USAEC Report c00-1569-11. 


\section{SIPAFE}

1 I. H. Nosanow

2 W. Zimmermann, Jr.

3

A. M. Goldman

W. V. Weyhmann

4 w. Mullin

5 E. Byokling

7

H. Horner:

8

C. M. Varma
Associate Profesaor

Asmociate Professor

Assistant Professor

Assistant Profes\$

Research Associate

Reselarch Associate

Research Associate

Research Associate

\section{GRADUATE STUDENTS}

L. Abad

J. Aslam

พ. Gutheil

R * Houghton

S. Kral

P, Kreisman

A. Menard

R. Murphy

W. Praty

B. Sabo

F. Schaer

c. Smith

I. ivinarma:

$\boldsymbol{J}$. Yanez
Research Assistant

Research Assistant

Research Asgistant

Research Assistant

Research Assistprit and NSF Fellow

NASA Trainé

Regearch Aasistant:

Research Assistant

masia Trainee

- Research Assistant

NSF Trainee

Research Assiztant

- Research Assistant

Ford Foindation Fellow, Univ. of ‘.. conception 
1. John 'simon Guggeheim Fellow 1966-1967, on leave academic year 1966-1967 at University of Utrecht, the Netherlands.

2. NSF Senior Postdactoral Fellow $1967 \% 1968$, will be on leave acadenic year 1967-1968 at The Technical University of Helsinki.

3. Alfred P. Slọn Felilow.

4. Present address: University of Massachusetts, Amherst, Mass.

5. Preaent address: University of oulu, Oulu, Finland.

6. Requirements for $\mathrm{Ph} . \mathrm{D}$. completed.in August, 1966 , present address: Los Alamos Scientific Laboratory, Los Alamos, New Mexico.

7. Started 16 September 1967.

8. Started 16 september 1967.

9. Terminated 15 september 1967. 


\section{BIBLIGGRAPHY}

5. c00-1569-5 "Current-Carrying states of Weakly Coupled Superconductora" (A.. M. Goldman and P. J. Kreismen) To be pub1.lghed. in the Proceedings of LT-10, Moscow.

6. c00-1569-6 mobservation of Quantized circulation in Superfluid Helium" (S. C. Whitmore, doctoral dissertation):.

7. C00-1569-7 Meissner Effect: and Vortex Penetration in Wide, High-Current. Josepheon :Junctione" (A. M. Goldman and .P. J. Kreisman). Abstract of paper presented at the Chicago Meeting of the APS, . 1967.

B. c00-1569-8 "Critical Indices of the Eambda Transition" (E. Byckling) . Submitted to Phylitics Letters.

9. c00-1569-9 "The Effect of Pressure on the Lifetime of Negative Ions Trapped in Rotating Liquíd Helium II" (W. P. Pratt and W. Zimemermann, Ir.). Abstract pregented at the APS Society, April 1967. 
10. c00-1569-10 Meissner Effect and Vortex Penetration in Josephson Junctions" (A. M. Goldmen and P. J. Kreisman). Physical Review, to be published.

11. Co0-1569-11 "Liquid Helium Green Functions near the Lambda Transition" (E. Byckling). Submitted to The Physical Review.

12. c00-1569-12 "Flow of Superfluid Helium in a Porpus Medium" (J. B. Mehl and w. Zimmermann, Jr.) Submitted to The Physical Review.

13. co0-1569-i3 "Observation of Quantized circulation in Superfluid

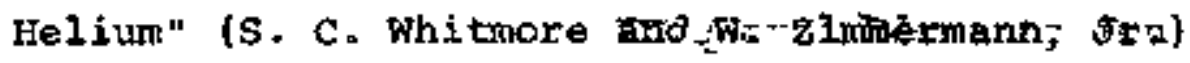
Submitted to The Pḩ̧sical Review.

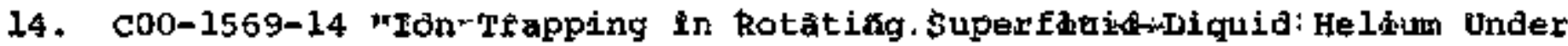
Pressure" (W. P. Pratt) Doctoral Dissertation.

15. C00-1569-15 "Progress Report (1967).

16. C00-1569-16 "A Critique of the Saunderg Theory of Solid ${ }^{3} \mathrm{He}$ at $0^{\circ} \mathrm{K}^{+}$. (w. J. Mullin) submitted to Physics.

17. Co0-1569-17 "Theory of Quantum Crystals" (L. H. Nosanow, W. J. Mullin and J. H. Hetherington) Presented at the $10^{\text {th }}$ Interis, 10, national Conference on Low Temperature Physics. 